\title{
,Weises' Lachen als Erziehungsmittel. Ricarda Huchs Novelle Teufeleien
}

\author{
'Wise' laugth as an educative measure. \\ Ricarda Huch's novella Teufeleien
}

\begin{abstract}
The article is an attempt to present the most important features of Ricarda Huch's writing found in the early stage of her literary creativity. From the methodological point of view, the basis for the research are the author's biography and her inveteracy in the history of early German Romanticism. According to the early romantics, and particularly Novalis, Huch aspired to achieve a kind of synthesis between historical facts and the way of perceiving reality by a writing artist, who, while thinking about his contemporaries, is guided by the pedagogical idea whose primary goal is to observe the principles of humanitarianism in the society. Keywords: Ricarda Huch, historicism, early romanticism, reformation, persecution of witches, humanitarianism.
\end{abstract}

Im Jahre 1895 erscheint im schweizerischen „Sonntagblatt des Bund“ die Novelle Eine Teufelei. Nachgelassene Papiere des Staatsschreibers Potzmaterle $^{1}$ von Ricarda Huch (1864-1947). Auf den ersten Blick scheint Huchs Fabel unkompliziert und wenig anspruchsvoll der Vorstellungswelt des möglichen Lesers gegenüber zu sein: In der alten Eidgenossenschaft geistert der Teufel herum und treibt sein Unwesen. Die Bewohner eines kleinen, abgelegenen Bergstädtchens fürchten sich vor der auf der Wiese tanzenden

1 Im Folgenden wird der Titel in Kurzform als Teufeleien angegeben. 
und unschuldige Mädchen verführenden Verkörperung des Bösen. Die teuflische Gestalt erweist sich als mächtig genug, um sogar vor den geistlichen Würdenträgern standzuhalten. In einem letzten Kraftakt versucht sich die Kleinstadtbevölkerung vor dem Teufel zu retten, indem ein Teufelsbeschwörer bestellt wird, der jedoch versagt, und es scheint, dass es für die Bewohner keine Rettung mehr gibt...

Wenn die äußere Handlung auch relativ einfach nacherzählt werden kann, so gibt es im intertextuellen Bereich Themenfelder und Erzählstrukturen, die Konnotationen, Verbindungen und Überleitungen ermöglichen, die dazu führen, den Positionierungen der Autorin Ricarda Huch, sich selbst wie auch dem humanen Verhalten der Menschen in der Gesellschaft gegenüber, gerecht zu werden. Somit hat dieser Beitrag das Ziel, Einblicke in Huchs literarische Konstruktionen des, weisen ' Lachens zu gewähren und zugleich die von ihr als Literatin gesetzten Zeichen für eine mögliche Umerziehung der Denk- und Betrachtungsweise der Realität zu erkennen.

\section{Huch als eine an der Romantik orientierte Intellektuelle ihrer Zeit}

Die Autorin Huch verstand auch sich selbst als zur Zielgruppe ihrer Leser gehörig, weil sie unermüdlich an sich selbst als Intellektuelle arbeitete und nach neuen Entwicklungsmöglichkeiten für ihre Dichtkunst suchte. Die Literatin, Historikerin und Denkerin Ricarda Huch, die heute zu den wenig gelesenen und rezipierten Persönlichkeiten der deutschen anspruchsvollen Literatur- und Geistesgeschichte gehört, verstand den Menschen als Individuum, das irrt und von dem rechten Weg der humanen Vorsätze abgleitet, aber zugleich durch durchdachtes und geschicktes Hinlenken in der Lage ist, sich, an positiven Beispielen aus der Literatur und Geistesgeschichte orientierend, leiten zu lassen, um unter den Voraussetzungen der allgemeinen Humanität zu einem nützlichen Glied der Gesellschaft zu werden. Dies bedeutet wiederum, zum Wohl der Gesellschaft und der allgemeinen Staatlichkeit beizutragen. Diese Lebenseinstellung vertrat Huch in ihrem Handeln als verantwortungsvolle Denkerin und Dichterin, wovon ihr vielseitiges OEuvre ein Zeugnis ablegt.

In Huchs Gesamtwerk lassen sich zwei Phasen unterscheiden, die zum einen ihre Reifung als Dichterin zeigen, und zum anderen ihre Sicht auf die Verantwortung als Intellektuelle ihrer Zeit und den Mitmenschen gegenüber präsentieren. Die erste Phase ihres Schaffens ist von der intensiven Auseinandersetzung mit der deutschen Romantik, insbesondere der Frühroman- 
tik, erfüllt. In der zweiten Phase, um die Jahrhundertwende, zeichnet sich Huchs Begeisterung für die Poetisierung der Geschichte ab. Der Impuls für Huchs Begeisterung für die deutsche (Früh)Romantik ist in ihrer Suche nach einem Modell für das Ausleben der künstlerischen Freiheit zu sehen. Huch fasst ihre Überlegungen zur Romanik in dem 1899 veröffentlichten ersten Teil ihrer literaturhistorischen Studie unter dem Titel Blütezeit der Romantik zusammen. Im Mittelpunkt ihrer Betrachtung steht der romantische Mensch als das neue Künstlerideal:

Eine Schar junger Männer und Frauen erobernd über die breite träge Masse Deutschlands. Sie kommen [...]: abenteuerlich, siegesgewiß, heilig erfüllt von ihrer Sitte und ihrem Leben, mit übermütiger Verachtung die alte morsche Kultur über den Haufen werfend. ${ }^{2}$

Diese jungen Nein-Sager erkennen die Erneuerungsnot, verspüren Erneuerungswillen und -sehnsucht. Zugleich setzen sich für den geistigen, ästhetischen und moralischen Aufbruch des Menschen und der Gesellschaft ein. Das menschliche Ich erwacht zum kühnen Selbstbewusstsein und soll tätig werden. Die Frühromantiker vergleicht Huch mit den „indiensuchende[n] Träumer[n], [...] [die] ihre Seele aus nach dem uralten Wunderlande [sandten], von dem die Märchen der Vorzeit erzählten“3. Folge dieser geistigen Suche ist ein Mentalitätswandel, der in der Lust am Geheimnisvollen und Wunderbaren gründet und zugleich den rationalistischen Geist zurückdrängt. Der Glaube an die menschliche Vernunft wird durch das Schicksalhafte $^{4}$ und das Geheimnisvolle ${ }^{5}$ ersetzt, was wiederum das Interesse auf menschliche innere Erlebnisse lenken kann.

Denn nicht [...] irgendein wunderbares Traumland war es, sondern in ihnen selbst öffnete sich das unendliche Nachbarland ihres Geistes, die entgegengesetzte Scheibe

2 Ricarda Huch, „Die Gebrüder Schlegel“, in Ricarda Huch, Gesammelte Werke, Bd. 6: Literaturgeschichte und Literaturkritik: Blütezeit der Romantik, hrsg. v. Wilhelm Emrich (Köln: Verlag Kiepenheuer \& Witsch, 1969), 23.

3 Ricarda Huch, „Apollo und Dionysos", in Ricarda Huch, Gesammelte Werke, Bd. 6: Literaturgeschichte und Literaturkritik: Blütezeit der Romantik, hrsg. v. Wilhelm Emrich (Köln: Verlag Kiepenheuer \& Witsch, 1969), 90.

4 Friedrich Schlegel schreibt im Januar 1792 über Novalis: „Das Schicksal hat einen jungen Mann in meine Hände gegeben, aus dem alles werden kann." Friedrich. Schlegel, Briefe an seinen Bruder August Wilhelm, hrsg. v. Oskar F. Walzel (Berlin: Verlag Speyer \& Peters 1890), 34 .

5 Novalis fordert den Spekulationsgeist: „Indem man dem Gemeinen einen hohen Sinn, dem Gewöhnlichen ein geheimnisvolles Ansehen, dem Bekannten die Würde des Unbekannten, dem Endlichen einen unendlichen Schein gebe, so romantisiere ich es.“ Novalis, „Die Welt muss romantisiert werden", in Theorie der Romantik, hrsg. v. Herbert Uerlings (Stuttgart: Reclam-Verlag, 2000), 51-52. 
des beseelten Planeten, wie einer von ihnen die verhüllte Hälfte des mit sich selbst unbekannten Menschen nennt, hatte sich ihnen zugewendet. ${ }^{6}$

Die Literaturhistorikerin Huch erkennt die Zweifel der jungen Erneuerer an ausschließlicher Vernunfterkenntnis, die dazu führt, dass das menschliche Individuum seine ursprüngliche Ganzheit, bestehend aus einer Harmonie zwischen der geistigen und körperlichen Sphäre, verliert. Die Frühromantiker sprechen von der „Doppelerscheinung des Ich"7. Zugleich findet Huch in der künstlerischen Tätigkeit und Wirkung der jungen Romantiker eine Möglichkeit für eine Zusammenführung dieser Polarität zwischen der Realität und dem inneren Erleben, und zwar in der Verkörperung von Novalis, der für Huch zum Gewährsmann der frühromantischen Strömung wird. Novalis gehörte nach Huch:

eben nicht zu jenen Idealisten, die die Augen an den Sternen hängend mit den Füßen durch den Sumpf waten, im Gegenteil pflegte er nach Art des guten Realisten mehr zu leisten, als er versprach, indem seine Äußerungen über sich selbst sich immer nur mit dem Nächstliegenden beschäftigten, war er sich erlebt hatte und wofür er einstehen konnte. ${ }^{8}$

Seine „leichten Füße" ${ }^{\text {" }}$ tragen ihn in die jenseitige Sphäre seines Inneren, aber lassen ihn zugleich im Bodenständigen verankert bleiben, was ihn wiederum lebensfähig macht. ${ }^{10}$ Dieses an Novalis erprobte Modell eines Künstlers, der die Realitätsverankerung nicht verloren und dennoch die Wirklichkeit ästhetisch aufgefasst habe, wird für Huch zum nachahmenswerten Lebensmotto als schreibend-schaffende Künstlerin und zur Quelle für die Verwirklichung des Humanitätsideals.

\section{Huchs Hinwendung zu geschichtlichen Stoffen}

Der Lebens- und Kunstauffassung von Novalis treu, suchte Huch um die Jahrhundertwende nach einer Erweiterung ihres poetischen Tätigkeitsfeldes und sah in der Geschichtsschreibung ein Potenzial für ihren künstleri-

6 Huch, „Apollo und Dionysos“, 90.

7 Huch, „Apollo und Dionysos“, 93.

8 Huch, „Apollo und Dionysos“, 76.

9 Huch, „Apollo und Dionysos“, 77.

10 Auch einer der prominentesten Literaturkritiker, Marcel Reich-Ranicki, verwies auf die Begeisterung Huchs für Novalis' praktische Lebenssicht, die ihn nicht daran hinderte zugleich ästhetisch die Welt zu erkennen. Vgl. Marcel Reich-Ranicki, „Ricarda Huch, der weiße Elefant," in Ricarda Huch. Studien zu ihrem Leben und Werk. Aus Anlaß des 120. Geburtstages (1864-1984), Bd. 1, hrsg. v. Hans-Werner Peter (Braunschweig: PP-Verlag GmbH, 1985), 4. 
schen Ausdruck. Als promovierte Historikerin entdeckte Huch für sich eine neue Art der Vermittlung von historischem Geschehen. In der um die Jahrhundertwende von den großen Persönlichkeiten der deutschen Geschichtsschreibung, wie Leopold Ranke (1795-1886), angeregten Diskussion um die Forschungswege des Historikers ${ }^{11}$ setzte sich Huch für eine Geschichtsschreibung ein, die zwar faktenorientiert arbeitet, aber zugleich vor die Fakten den agierenden Menschen der Geschichte stellt und bestimmte Persönlichkeiten als Hauptakteure des Weltgeschehens betrachtet. Sie entwickelte über Jahrzehnte ihres Schaffens ein Modell für den Be- und Erschreibungsweg von Geschichte. ${ }^{12}$ Eine Möglichkeit für die Umsetzung dieses Vorhabens eines an der Ästhetisierung der Geschichte interessierten Historikers sah Huch in der Vergangenheit der alten Eidgenossenschaft. Erste Erfahrungen mit der Geschichtsforschung machte Huch während ihres Geschichts-, Literatur- und Philosophiestudiums an der Zürcher Universität in den Jahren 1888-1891 sowie während ihrer Arbeit als Bibliothekarin in der Zürcher Stadtbibliothek. Als für die alten Handschriften zuständige Bibliotheksmitarbeiterin vertiefte sich Huch in die geschichtliche Vergangenheit der Schweiz. Es scheint, dass insbesondere die Reformationszeit in der Schweiz sie angesprochen hat, als eine Zeit voller innerer Widersprüche und Kämpfe der Fortschrittlichen gegen die Konservativen, wobei für Huch die Auseinandersetzung selbst zum Zeichen der Erneuerung wurde. In der Überzeugung des Zürcher Reformators Ulrich Zwingli (1484-1531) ${ }^{13}$, dass der Mensch

11 Leopold von Ranke vertrat als Historiker den historischen Realismus in der wissenschaftlichen Forschung und der Darstellung von Forschungsergebnissen. Huch vermisste bei Ranke: „das unterirdische Rauschen der übermenschlichen Mächte,“ die Betrachtung des Vergangenen aus mehreren Perspektiven. Ricarda Huch, „Frühling in der Schweiz“, in Ricarda Huch, Erinnerungen an das eigene Leben, Nachw. v. Bernd Balzer (Köln: Ullstein Werkausgaben, 1983), 209.

12 Vgl. auch Hayden Withe, „Fikcjonalność przedstawień opartych na faktach”, übers. v. Dorota Kołodziejczyk, in Hayden Withe, Proza historyczna, hrsg. v. Ewa Domańska (Kraków: Universitas, 2009), 87-105.

13 Sich an den Grundsätzen des Humanismus orientierend, vertiefte sich Ulrich Zwingli in das Studium der Bibel und der theologischen Schriften. Dadurch gewann er eine neue Weltsicht in Bezug auf Glaubensfragen. Als er am 1. Januar 1519 seinen Posten als Leutpriester am Zürcher Großmünster übernahm, wurde die Bibelexegese in seinen Predigten zum Mittelpunkt des Gottesdienstes. Sein religiöses Bekenntnis wurde insbesondere durch seine Pesterkrankung 1519 und die darauffolgende Genesung, die er als Zeichen Gottes betrachtete, gestärkt. Während der ersten Disputation vom 23. Januar 1523 konnte Zwingli seinen Standpunkt bezüglich der Ablehnung des Sold- und Pensionswesens und der Übertretung des Fastengebots erfolgreich rechtfertigen. Folge war, dass die Reformation in Zürich bis zum Jahr 1525 vollständig durchgeführt war und sich auch auf Basel, Schaffhausen, Bern und Sankt Gallen erstreckte. Vgl. Christian Schutt, Hg., Chronik der Schweiz (Zürich: Ex Libris Verlag/ Chronik Verlag, 1987), 225-229. 
sich einzig am Wort des Erlösers Jesus Christus zu orientieren habe, um zur Seligkeit zu gelangen, ohne die vermittelnde Rolle der Kirche ${ }^{14}$, fand Huch eine Bestätigung für ihre Überzeugung von der Entscheidungsfreiheit des menschlichen Individuums und seiner Kraft, sich im Handeln dem Guten zuzuwenden. Huch ging auf die Reformation in der Schweiz nicht näher ein. Für sie als an der Geschichte sich orientierenden Literatin war es viel ansprechender, die die reformatorischen Auseinandersetzungen begleitenden dynamischen Fortschrittsprozesse zu beobachten, die wiederum für Huch zum Zeichen des voranschreitenden Erwachens des menschlichen Selbstbewusstseins wurden.

Zu einem geschichtlichen Gewährsmann für diese Zeit wurde für die Historikerin Huch Johann Jakob Wick (1522-1588) ${ }^{15}$, der seit 1557 zum zweiten Archidiakon am Zürcher Großmünster und zum Anhänger der Reformationsbewegung in der Schweiz unter Ulrich Zwingli wurde. Wick hinterließ der Nachwelt eine Handschriftensammlung, die sog. Wickiana, welche die bedeutendste Nachrichtensammlung von Einblattdrucken und illustrierten Flugblättern des 16. Jahrhunderts (aus der Zeitspanne zwischen 15591588) darstellte. Die Bibliothekarin Huch betreute diese Sammlung und setzte sich mit ihr auseinander. Ergebnis dieser Arbeit war Huchs Studie über Die Wicksche Sammlung von Flugblättern und Zeitungsnachrichten aus dem 16. Jahrhundert, die im „Neujahrsblatt der Stadtbibliothek Zürich“ für das Jahr 1895 erschien, und die Huch als ein Abschiedsgeschenk an die Bibliothek betrachtete. In ihrem einleitenden Wort zu dieser Studie wendet sich Huch an den Leser und macht ihm deutlich, dass seine Vorstellungskraft gefordert werde. Erklärend schreibt sie: Wenn man die alten archivalischen Berichte studiert, „glaubt [... man] mitten in eine fremde Welt hineinversetzt zu sein. Es wird einem zu Mute wie einem, der eine ferne Gegend lange aus den Schilderungen der Bücher kannte und sie nun plötzlich vor seinen Augen sieht" ${ }^{\prime \prime}$. Die Autorin Huch hat sich zum Ziel ihrer historisch orientierten Darstellungen gesetzt, ihren Rezipienten diese fremd angehauchte Welt, die

14 Schutt, Chronik der Schweiz, 225.

15 Johann Jakob Wick genoss eine klassische Ausbildung in dem Kloster Kappel. Anschließend studierte er Theologie in Tübingen und Marburg. Nach dem Studium wirkte er als Pfarrer in Zürich-Witikon. 1552 wurde er zum Spitalpfarrer in Zürich berufen und 1557 avancierte er zum zweiten Archidiakon am Zürcher Großmünster. Wick wurde zum Anhänger der Reformation unter Ulrich Zwingli. Zugleich wurde er durch seine Wickiana zum Stadt- und Zeitchronisten. Vgl. Franz Mauelshagen, „Wick, Johann Jakob“, in Biographisch-Bibliographisches Kirchenlexikon, Bd. 17 (Herzberg: Traugott Bautz Verlag, 2000), 1536-1540.

16 Ricarda Huch, „Die Wicksche Sammlung von Flugblättern und Zeitungsnachrichten aus dem 16. Jahrhundert", in Ricarda Huch, Gesammelte Werke, Bd. 9: Geschichte 1, hrsg. v. Wilhelm Emrich (Köln: Verlag Kiepenheuer \& Witsch, 1968), 264-265. 
sich der ausschließlichen Vernunfterkenntnis entzieht, in der auch realitätsferne Wesen auftreten können, „zugänglicher“ zu machen, und diese Welt mit Hilfe von poetischen Bildern zu entschlüsseln. Daher kleidete Huch einige Ereignisse aus der Zeit der Reformationszeit in der Schweiz in eine literarische Erzählung ein. Auf diese Weise konnte der historische Stoff spannend erzählt werden und die Leser anspornen, über ihr eigenes Verhalten nachzudenken.

Das Thema, mit dem sich Huch die Möglichkeit eines Diskurses mit ihrer Zeit erhoffte, waren die historischen Überlieferungen über Teufelserscheinungen und Hexenumtriebe in der alten Eidgenossenschaft im 16. Jahrhundert. Im Jahre 1545 wurden in der Schweiz die Hexenverfolgungen fortgesetzt und erreichten ihren Höhepunkt. Der Glaube an Hexen und Zauberer beschränkte sich nicht nur auf den ungebildeten Teil der Bevölkerung, es gab auch angesehene Theologen wie der Genfer Reformator Johannes Calvin (1509-1564), die an das Wirken übernatürlicher Kräfte glaubten und sich persönlich für harte Strafen für die der Zauberei Beschuldigten einsetzten. Auch der Basler Theologe Oswald Myconinus (eig. Geisshülser, 1488-1552) glaubte an die sogenannte Genfer Pestverschwörung, wobei er in seinem Brief vom 27. März 1545 an Johannes Calvin berichtet:

Obwohl fünfzehn Weiber verbrannt, einige Männer noch grausamer hingerichtet wurden, einige im Kerker selbst den Tod suchen, noch fünfundzwanzig gefangen gehalten werden, hören sie doch nicht auf, jeden Tag die Haustürschlösser mit ihren Salben zu bestreichen. Sieh, in welcher Gefahr wir schweben... ${ }^{17}$

Der Aberglaube, sogar von den Gebildeten verbreitet, bildete für Huch ein interessantes Themenfeld, um den Menschen, auch ihren Zeitgenossen, mit seinem engstirnigen Denken und mit dem verstandesmäßig Fremden und Unerklärbaren unmittelbar zu konfrontieren. Diese scheinbare Angst vor dem Fremden und Unerklärbaren macht den Menschen entwicklungsunfähig und lässt die negativen Charaktereigenschaften aufkommen.

\section{Die Novelle Teufeleien}

Ihren Zeitgenossen wollte Huch aus solch einer Lage buchstäblich retten, indem sie ihn wachzurütteln beabsichtigte und die folgende historisch überlieferte Geschichte, die sie in die Gattungsform einer Novelle Eine Teufelei. Nachgelassene Papiere des Staatsschreibers Potzmaterle kleidete, erzählte. Eine Bestätigung für ihr erzählerisches Vorhaben, mit Hilfe von geschichtlicher Überlieferung die überzeitlichen Anliegen der Menschen anzusprechen,

17 Schutt, Chronik der Schweiz, 244. 
fand Huch in der Zusprache des Herausgebers des Berner Magazins „Sonntagsblatt des Bund“, Josef Viktor Widmann, der zugleich ein unermüdlicher Förderer des literarischen Talents von Huch war. Widmann bemerkte in seinem Brief vom 20. 04. 1895 an die Autorin, dass es durchaus möglich sei, aus geschichtlichen Überlieferungen Inspirationen für das poetische Werk zu schöpfen und die Aktualisierung dieses Stoffes zu finden. ${ }^{18}$ Widmann sprach Huchs Gabe an, literarische Personen zu kreieren, die dem wirklichen, erstarrten Leben nahe seien und zugleich den Leser in eine Welt voll Phantasie, Dynamik, Widersprüche und Überraschungen entführen, in der er, aus den konventionellen Zusammenhängen entrissen, dank seiner Vorstellungskraft mit einer neuen künstlerischen Realität konfrontiert werde und sich in dieser zurechtfinden müsse.

In der bei Widmann veröffentlichten Novelle Teufeleien geht Huch gezielt auf eine von Wick überlieferte Geschichte aus Zug ein, die sich um das Jahr 1574 zugetragen haben soll. Wick berichtete, dass wegen des sich umtreibenden Teufels das Vieh massenhaft starb und die Ratsherren sowie Geistlichen in ihrer Ausweglosigkeit beschlossen, Hilfe bei einem Teufelsbeschwörer zu suchen:

Am hohen Donnerstag saß der Teufelsbeschwörer in Zug im Wirtshaus zu Krone und nahm das Nachtessen ein; hernach, sagte er, wolle er mit dem vier Teufeln dran [...] Der Teufelsbeschwörer springt mitten unter sie, und das war das letzte, was [seine zwei Begleiter] von ihm sahen. ${ }^{19}$

Zu einem Vermittler zwischen Phantasiewelt und realer Welt wird in Huchs Text der auktoriale Ich-Erzähler. Er konfrontiert den Leser mit unterschiedlichen Reaktionen der Handlungsfiguren auf das Erscheinen von Teufel- und Hexenwesen. Dieser Ich-Erzähler wird zu der den Handlungsverlauf gestaltenden, strukturierenden und die Zusammenhänge erklärenden Erzählinstanz und zugleich zum Sprachrohr der Autorin selbst.

Der Ich-Erzähler wird als Stadtchronist unmittelbarer Zeuge einer Anhörung, in der Trud, der Tochter eines angesehenen Seckelmeisters, eine intime Verbindung mit dem Teufel nachgesagt wird, woraufhin man sie nötigt, Stellung zu diesen Anschuldigungen zu nehmen. Huch konfrontiert hier die Welt der Realität mit der Welt von außernatürlichen Erscheinungen und Mächten. Die nachgezeichnete Szene enthüllt die Mentalität der Beteiligten, die unterschiedliche Schichten der damaligen Gesellschaft vertreten. Die

18 Jutta Bendt und Karin Schmidgall, Hg., Ricarda Huch 1864-1947. Eine Ausstellung des Deutschen Literaturarchivs im Schiller-Nationalmuseum Marbach (Marbach am Neckar: Deutsche Schillergesellschaft, 1994), 64.

19 Ricarda Huch, „Die Wicksche Sammlung von Flugblättern und Zeitungsnachrichten aus dem 16. Jahrhundert", 292. 
oberste städtische Instanz - der Stadtrat -, der zugleich auch die Justiz vertritt und als Gemeindeexekutive tätig ist, wurde darüber benachrichtigt, dass

auf der großen Wiese, des Seckelmeisters Hause gegenüber, [...] man in der Nacht allerlei Ungewöhnliches und Unrichtiges wahrgenommen habe. Es habe etwas gerauscht und gemunkelt, hüpfende Lichtlein habe man gesehen, dazu Gestalten, die menschenähnlich erschienen wären, ja, es wollte sogar einer die Tochter des Seckelmeisters erkannt haben. ${ }^{20}$

Dieses Gerücht wird zum Gegenstand einer rechtmäßigen Gerichtsanhörung und für die Autorin Huch zur Gelegenheit, über die Bedeutung des Gerichtswesens in einer (scheinbaren) staatlichen Demokratie zu schreiben. Huch macht deutlich, dass der Stadtrat sich der Öffentlichkeit als gewissenhaft und pflichtbewusst zeigen und daher auch um jeden Preis die Wahrheit ermitteln wollte, um somit seiner Aufgabe gemäß zum Wohlergehen der Gemeinschaft und zur Wahrung des Gesetzes beizutragen. Der Ich-Erzähler besitzt die Gabe, hinter diese scheinbar heile, gerechte Welt zu blicken, und überrascht sieht er, dass „diese Herren dem Volke gegenüber doch zusammen wie Schwammpilze [hielten] und [den Seckelmeister] insgeheim vor dem Vorgang in Kenntnis [setzten], mit Andeutung, wenn eine verschwiegene Sache vorliege, wolle man sorgen, daß nichts davon unter die Leute komme“21. Huch teilt die Erkenntnis ihres Ich-Erzählers mit und prangert die Verlogenheit und Doppelmoral der scheinbaren, demokratisch bestellten Gemeinschaftsvertreter an. Zum Repräsentanten der „strengen [örtlichen] Gerechtigkeit"22 wird der Bürgermeister, der „dem Ruhme nach[trachtete], mit jenem alten Römer namens Brutus verglichen zu werden“23. Im Laufe der Handlung wird der wahre Charakter dieser öffentlichen Person entblößt, wenn der Rezipient bedenkt, dass der antike Brutus nicht dem Gerechtigkeitssinn folgte, sondern sich selbst als die höchste Rechtsinstanz zu positionieren versuchte. Es scheint, dass auch diese um jeden Preis erreichte gesellschaftliche Stellung den Bürgermeister beglücken würde. Die Vertreter der Justiz werden daher als korrumpierte, verlogene, skrupellose und auf das eigene Wohlergehen bedachte Personen der Öffentlichkeit vorgeführt, die falsch genug seien, um vorzugeben, an die Verkörperung des Teufels zu glauben, damit ihr Ansehen in der Gemeinschaft gerettet werde.

Zur zweiten Zielscheibe der Huch'schen Kritik werden in der Novelle die Kirchenvertreter und somit auch die Praxis der Glaubensausübung. Huch

20 Ricarda Huch, „Teufeleien“, in Ricarda Huch, Gesammelte Werke, Bd. 4: Der Fall Deruga, Der wiederkehrende Christus. Romane. Sämtliche Erzählungen, hrsg. v. Wilhelm Emrich (Köln: Verlag Kiepenheuer \& Witsch, 1967), 529.

21 Huch, „Teufeleien“, 529.

22 Huch, „Teufeleien“, 530.

23 Huch, „Teufeleien“, 530. 
führt in die Handlung zwei Geistliche ein, die vom Stadtrat um Rat und Unterstützung gebeten wurden. Diese sind jedoch kein Beispiel für ein harmonisches Glaubensbekenntnis, weil es sich hier um zwei miteinander zerstrittene und verfeindete Priester, einerseits den Pfarrer der Blasius- und andererseits den der Anastasiuskirche, handelt. Sie sollen darüber entscheiden, ob der „Teufel ganz unschuldige Menschen anfalle, oder ob er sich nur an solche mache, von denen er wisse, daß sie seinen Verführungen früher oder später zugänglich sein werden"24. Mit dieser Akzentuierung leitet Huch eine Auseinandersetzung zwischen den Anhängern des vorreformatorischen und des reformierten Glaubens ein.

Der Autorin Huch geht es nicht um einen theologischen Diskurs in Bezug auf das Teufels- oder Hexenwesen, auf das sie nur in Umrissen eingeht. Ihre erzählerische Absicht tendiert zu einer Verortung und Verdeutlichung von menschlichen Verhaltensweisen. Zum Gewährsmann der für den erzählerischen Fortgang notwendigen Verdeutlichung des Hexenwesens wird für Huch nicht der Schweizer Reformator Ulrich Zwingli, sondern Martin Luther, an dessen Lehre Huch als überzeugte Protestantin sich selbst orientierte. ${ }^{25}$ Eine Ursache dafür liegt wohl in der Tatsache, dass Zwinglis Vorstellung von Hexen nicht so recht in Huchs erzählerisches Konzept passte. Für Zwingli sind nicht die Hexen die vom Bösen heimgesuchten weiblichen Gestalten, es sind vielmehr die Vertreter der Institution Kirche, die im Bund mit dem Teufel stehen und somit das Wohl der Kirche und den rechten Glauben bedrohen und sogar zerstören. ${ }^{26}$ Anders für Martin Luther, der durchaus das Wesen der Hexen als vom Teufel besessene weibliche Gestalten erkannt zu haben glaubte. ${ }^{27}$ Huch versuchte Luthers Verständnis von Teufel und Hexen modern zu deuten und somit auch den Reformator für ihr Zeitalter zu rehabilitieren. In Huchs Teufeleien ist der Teufel durchaus anwesend und bedroht unmittelbar den Menschen, also seine Seele und seinen Leib, genauso wie

24 Huch, „Teufeleien“, 532.

25 Gabriela Jelitto-Piechulik, „Ricarda Huchs Lutherprojektionen,“ in Die Reformation 1570. Zwischen Gewinn und Verlust, hrsg. v. Cezary Lipiński, und Wolfgang Brylla (Göttingen: Vandenhoeck \& Ruprecht, 2020), 167-178.

26 Zwingli wandte sich in seiner Auslegung und Begründung der Thesen 1523 kritisch gegen die kirchlichen Lehre über das Fegefeuer und erhob Anschuldigungen gegen die Kirchenvertreter, die er als Hexenmeister bezeichnete. Vgl. Christoph Scheidegger, „Hexen bei Zwingli“, in Reformierte Kirche des Kanton Zürich, https://www.zhref.ch/themen/ reformationsjubilaeum/allgemeine-informationen/huldrych-zwingli/zwingli-lexikonvon-a-bis-z-1/lexikon-h/hexen.

27 Vgl. Lorenz Sönke und H.C. Erik Midelfort. Hexen und Hexenprozesse. Ein historischer Überblick, historicum.net, Mai 15, 2018, https://www.historicum.net/purl/aj/. 
sich Luther vom Teufel bedroht fühlte und sich mit ihm gemessen hat. ${ }^{28}$ Den Teufel versteht Huch als die Macht des Bösen, welche die menschliche Existenz bedroht und ihn als humanes Wesen in den Abgrund treibt. Um ihre Sichtweise zu veranschaulichen, zeigt Huch anhand von zwei Betrachtungsmöglichkeiten des teuflischen Wesens, wie der Mensch sich dieser dämonischen Macht gegenüber zu verhalten habe. Wenn der Pfarrer der Blasiuskirche als Anhänger des vorreformatorischen Glaubens, die „Glaubenslehre mit dem Herzen" 29 auslegt, so vertritt der Priester der Anastasiuskirche die Überzeugung, dass der Mensch mit Geist und Körper seinem Glauben verpflichtet sei. Somit sei er davon überzeugt, dass sowohl Gott wie auch der Teufel als zwei Gegenpersonen in Erscheinung treten können. Der Blasiuspfarrer meint dagegen, dass der Mensch dem Teufel nur in seinem Inneren begegnen könne. Um dies zu beweisen, erklärt er sich bereit, es mit dem auf der Wiese herumgeisternden Teufel aufzunehmen, um nur durch seinen Glauben den Teufel zu bezwingen. Aber auch ihm gelingt es nicht, die Stadt vom Teufel zu befreien, weil er in seinem Glauben nicht stark genug ist. Der Anastasiuspfarrer legt Wert darauf, in einem „feierlichen Bußgottesdienst“" 30 Gott um Gnade zu bitten. Beide Geistlichen scheitern, weil sie nicht in der Lage sind, ihren Glauben auszuleben, um sich auf diese Weise der Macht der Bösen zu widersetzten.

Huch betont in ihrer Novelle, dass das Böse (oder das Prinzip des Bösen) gegenwärtig sei, und nur darauf warte, vom Menschen heraufbeschworen zu werden. Der Mensch glaube, dass er kraft des Bösen seine Gelüste/Triebe befriedigen könne, um somit seine Privatvorhaben zu verwirklichen. Das Prinzip des Bösen wird zum roten Faden der Novelle, der die einzelnen Handlungsetappen zu einer Einheit zusammenführt und damit den Erzählgang nach vorne treibt. Dieses Phänomen des zerstörerischen bösen Prinzips wird besonders am Beispiel des Vaters des beschuldigten Mädchens, des Seckelmeisters, deutlich. Er ist ein wegen seines Amtes ein geachteter Bürger der Stadt. ${ }^{31}$ Und er legt großen Wert auf sein öffentliches Ansehen.

28 Die wohl bekannteste Szene aus Luther Biographie ist sein angeblicher Wurf mit dem Tintenfass nach dem ihn zu verführen suchenden Teufel während seiner Zeit auf der Wartburg.

29 Huch, „Teufeleien“, 532.

30 Huch, „Teufeleien“, 547.

31 Das Amt des Seckelmeisters nahm seinen Ursprung im späten Mittelalter und war eine Institution der Städte und Länder zur Organisation der Haushaltsführung. Im Laufe der Geschichte wurde der Seckelmeister zu einem der einflussreichsten Beamten in den Kleinen Räten. Das Amt wurde für eine begrenzte Amtszeit bekleidet, dennoch brachte es wegen der zahlreichen Nebenleistungen viele Geldeinnahmen. Im 19. und 20. Jahrhundert wurde in der Gesetzgebung der Begriff Seckelmeister durch die Amtsbezeichnung Finanzdirektor oder Gemeindekassierer ersetzt. Vgl. Jolanda Leuenberger-Binggeli, „Die 
Es würde für ihn einen gesellschaftlichen Rufmord bedeuten, wenn es sich erweisen sollte, dass seine Tochter mit dem Teufel verkehre und womöglich eine Hexe sei. Die Widersacher des Seckelmeisters würden dagegen in Trud gerne eine Hexe sehen, weil sie einerseits den Seckelmeister vernichten würden und andererseits auch den guten Ruf von Trud, die für sie wegen ihrer geistigen Überlegenheit und äußerlichen Schönheit zur Bedrohung wird.

Die Autorin Huch lässt ihre Helden der Novelle jedoch nicht untergehen. Sie liefert sie zwar dem Bösen aus, aber verleiht ihren Figuren genug Kraft, die aus ihrer Besonnenheit und wahrer Menschlichkeit hervorgeht. Trud versteht ihre Überlegenheit den Ratsherren gegenüber und weiß diese zur rechten Zeit einzusetzen. Wenn sie bei der ersten Anhörung jeglichen Verkehr mit dem Teufel bestritten hatte, erkannte sie während der zweiten Befragung ihre prekäre Lage, weil „neulich [...] erst mehrere Hexen verbrannt worden [seien], das habe ihr im Sinn gelegen. “32 In dem Moment, in dem sich Trud zu retten versucht, erblickt sogar der Ich-Erzähler überraschend an ihr die äußeren Merkmale einer Hexe. Huch lässt jedoch ihre weibliche Protagonistin nicht im Stich. Aus eigener Kraft heraus will Trud ihre Unschuld beweisen, aber nicht durch die Preisgabe der Wahrheit, sondern sie wählt einen Weg, welcher der geistigen Auffassung des Stadtrats und der Stadtbewohner entspricht: Sie gibt zu, tatsächlich Opfer des Teufels zu sein und bittet die Geistlichen um Hilfe und Beistand während des gefürchteten nächsten nächtlichen Teufelsbesuchs.

Der Ich-Erzähler gewinnt Erkenntnisse, dass es sich bei diesen Teufelsbesuchen in Wirklichkeit um Truds Geliebten, den Junker Claudius, handelt, der aus der Stadt verbannt wurde, und dem die Seckelmeistertochter ihr Herz geschenkt hat. Der Ich-Erzähler verpflichtet sich, den beiden Liebenden Hilfe zu leisten, indem er sich zu einer Teufelsgestalt kostümieren lässt: „Ich selbst hatte eine geschnäbelte Larve, einem Papageien nicht unähnlich, große Fledermausflügel, welche ich auch bewegen konnte, dazu ein laubgrünes Kleid mit roten Läppchen besetzt. “33 Trud, der Ich-Erzähler und der Junker Claudius trieben kostümiert ihr Unwesen auf der besagten Wiese. Der Ich-Erzähler berichtet: Wir „fingen [...] an umherzutanzen, bald Hand in Hand, bald jeder für sich, schwenkten die Fackeln, deren jeder von uns eine mitgenommen hatte, und gebärdeten uns überhaupt so wunderlich und un-

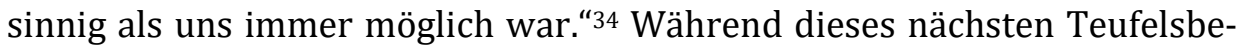

Berner Deutsch-Seckelmeister und ihre Standesrechnung“, Berner Zeitschrift für Geschichte und Heimatkunde 61 (1999): 153-186.

32 Huch, „Teufeleien“, 533.

33 Huch, „Teufeleien“, 545.

34 Huch, „Teufeleien“, 545 
suchs spielt sich vor den anwesenden Stadtbewohnern eine „Tragödie“ ab, der bestellte Teufelsbeschwörer versagt, Trud wird vom Teufel mitgenommen und bleibt verschwunden. Der am stärksten Betroffene ist jetzt der Seckelmeister, und dies nicht, weil er seine einzige Tochter verloren hat, sondern, weil er der Vater einer Teufelsbraut ist.

Es scheint, dass den Seckelmeister aus dieser prekären Lage nur der Junker Claudius retten kann, der wegen seiner Dienste für den "französischen Kriegsdienst [...] [und] unter Androhung, daß ihm die Todesstrafe treffen würde, wenn er sich auf unserm Gebiete wiederum blicken ließe" ${ }^{\prime 35}$, verbannt worden war. Der Junker findet die angeblich vom Teufel zurückgelassene Trud und nimmt sie bei sich auf. Die Sorge des Vaters richtet sich darauf, dass „ein Mädchen, das einmal in des Teufels Klauen gewesen sei, [...] keinen rechten Mann mehr [bekomme], noch wolle sonst irgend jemand mit ihr etwas zu schaffen haben." ${ }^{66}$ Und es erweist sich für den Vater als ein Gnadenakt, dass der Junker Claudius das vom Teufel geschändete Mädchen ehelichen will. Die einzige Figur der Handlung, welche die Zusammenhänge kennt und erkennt und in der Lage ist, das Geschehen zu überblicken und die Gefühle der handelnden Figuren nachzuvollziehen, ist der auktoriale Ich-Erzähler. Er entscheidet sich, den Liebenden zu helfen:

Indessen sagte ich, wie dem auch sei, wenn sie mit dem wahren Teufel im Bunde stände, würden mich keine Schätze der Welt vermögen, gemeinschaftliche Sache mit ihr zu machen, da aber in diesem Falle der Teufel nur ein Vorwand sei, wollte ich sie nicht im Stiche lassen. ${ }^{37}$

Wenn auch seine Entscheidung Notlügen nach sich zieht, so wird er zu einer Erzählinstanz, welche die Zusammenhänge der Handlung sinngemäß einordnet. Huch stilisiert ihn somit zu einem „weisen“ Narren, der seine Borniertheit zum Vorwand für wahre Menschenkenntnis und Nächstenliebe nutzt. Er versteht auch Truds Notlüge:

Was hätte ich denn tun sollen? Hätte ich sagen solle: Der Junker Claudius war auf der Wiese? Wäre mir etwas Besseres eingefallen, ihn zu retten, hätte ich das lieber vorgebracht, aber es kam mir nichts anders in den Sinn. ${ }^{38}$

Trud erweist sich als eine Frau, die stark genug ist, für ihre Liebe zu kämpfen und sich sogar in der Öffentlichkeit in den Verdacht der Verbindung mit dem Teufel zu bringen, als auf ihren Geliebten zu verzichten. Sie zeigt sich geistig den anderen Stadtbewohnern überlegen und mutig genug, für ihr persönliches Glück zu kämpfen. Um ihr Ziel zu erreichen, scheut Trud nicht

35 Huch, „Teufeleien“, 536.

36 Huch, „Teufeleien“, 556.

37 Huch, „Teufeleien“, 540.

38 Huch, „Teufeleien“, 537. 
davor zurück, ihren guten Namen zu opfern. Dabei schließt sie eine mögliche Niederlage nicht aus.

Diese Charaktereigenschaften der Erzählheldin erinnern an die Autorin Ricarda Huch selbst. In der von Trud ausgesprochenen Frage „Was hätte ich den tun sollen?" ${ }^{39}$, klingt auch die existentielle Fragestellung der Autorin selbst, die sie ihr Leben lang begleitet und von ihr wichtige Lebensentscheidungen abverlangt. Als junge Frau entscheidet sie sich bewusst für die verbotene Liebe zu ihrem Schwager Richard Huch, geht in die Schweiz, um sich hier universitär ausbilden zu lassen und in Zukunft den eigenen Lebensunterhalt als berufstätige Frau bestreiten zu können. Als Frau erkämpft sie sich die Unabhängigkeit und gestaltet ihr Leben autonom, bricht jedoch nicht mit den gesellschaftlichen Konventionen, sondern bewahrt für sich die geistige Freiheit als freie Schriftstellerin und Kunst schaffendes Individuum. Huchs Erkenntnisweg geht über einzelne Lebensstationen, die eine künstlerische Widerspiegelung in ihrem Werk finden. Huch als Frau und Persönlichkeit nimmt die Herausforderungen des Lebens an und wächst an diesen. Was nicht bedeutet, dass sie den für sich eingeschlagenen Weg nicht auch korrigieren musste, um ans angestrebte Ziel zu gelangen.

\section{Fazit}

Mit der Novelle Teufeleien versuchte Huch die ihr eigene Lebensdevise nachzuzeichnen und sich zugleich mit den Fragestellungen des menschlichen Daseins als Individuum und als Mitglied der Gesellschaft auseinanderzusetzen. Nie hat sie sich selbst oder den Menschen an sich als fertiges Produkt betrachtet, sondern als ein sich auf der Suche befindendes Wesen, das oft, religions- und geistesgeschichtlich gesehen, Irrwege eingeschlagen hat, aber später kraft der richtigen Entscheidungen, die in humanen Vorsätzen und im rechten Glauben beruhen, sich vor der vernichtenden Kraft des Bösen und somit vor der existentiellen Niederlage bewahren konnte. Die Fähigkeit, über die menschlichen Laster und Schwächen „weise“ lachen zu können, verstand Huch als die Pflicht eines Intellektuellen, der seine pädagogische Aufgabe ernst nimmt und seinen Mitmenschen zum wahren sozialgerechten Verhalten verhilft.

39 Huch, „Teufeleien“, 537. 


\section{References}

Bendt, Jutta, and Karin Schmidgall, eds. Ricarda Huch 1864-1947. Eine Ausstellung des Deutschen Literaturarchivs im Schiller-Nationalmuseum Marbach. Marbach am Neckar: Deutsche Schillergesellschaft, 1994.

Huch, Ricarda. "Die Wicksche Sammlung von Flugblättern und Zeitungsnachrichten aus dem 16. Jahrhundert." In Huch, Ricarda. Gesammelte Werke, vol. 9: Geschichte 1, edited by Wilhelm Emrich, 261-304. Köln: Verlag Kiepenheuer \& Witsch, 1968.

Huch, Ricarda. "Entpersönlichung." In Huch, Ricarda. Gesammelte Werke, vol. 7: Schriften zur Religion und Weltanschauung, edited by Wilhelm Emrich, 625-803. Köln: Verlag Kiepenheuer \& Witsch, 1968.

Huch, Ricarda. "Frühling in der Schweiz." In Huch, Ricarda. Erinnerungen an das eigene Leben. Afterword by Bernd Balzer, 176-243. Köln: Ullstein Werkausgaben, 1983.

Huch, Ricarda. "Teufeleien". In Huch, Ricarda. Gesammelte Werke, vol. 4: Der Fall Deruga, Der wiederkehrende Christus. Romane. Sämtliche Erzählungen, edited by Wilhelm Emrich, 529-574. Köln: Verlag Kiepenheuer \& Witsch, 1967.

Huch, Ricarda. Apollo und Dionysos. In Huch, Ricarda. Gesammelte Werke, vol. 6: Literaturgeschichte und Literaturkritik: Blütezeit der Romantik, edited by Wilhelm Emrich, 90-118. Köln: Verlag Kiepenheuer \& Witsch, 1969.

Huch, Ricarda. Die Gebrüder Schlegel. In Huch, Ricarda. Gesammelte Werke, vol. 6: Literaturgeschichte und Literaturkritik: Blütezeit der Romantik, edited by Wilhelm Emrich, 23-43. Köln: Verlag Kiepenheuer \& Witsch, 1969.

Jelitto-Piechulik, Gabiela. "Ricarda Huchs Lutherprojektionen." In Die Reformation 1570. Zwischen Gewinn und Verlust, edited by Cezary Lipiński, and Wolfgang Brylla, 167-178. Göttingen: Vandenhoeck \& Ruprecht, 2020.

Leuenberger-Binggeli, Jolanda. "Die Berner Deutsch-Seckelmeister und ihre Standesrechnung." Berner Zeitschrift für Geschichte und Heimatkunde 61 (1999): 153-186.

Mauelshagen, Franz. "Wick, Johann Jakob." In Biographisch-Bibliographisches Kirchenlexikon, vol. 17, 1536-1540. Herzberg: Bautz Verlag, 2000.

Novalis. "Die Welt muss romantisiert werden." In Theorie der Romantik, edited by Herbert Uerlings, 233-266. Stuttgart: Reclam-Verlag, 2000.

Reich-Ranicki, Marcel. „Ricarda Huch, der weiße Elefant.” In Ricarda Huch. Studien zu ihrem Leben und Werk. Aus Anlaß des 120. Geburtstages (18641984), vol. 1, edited by Hans-Werner Peter, 1-10. Braunschweig: PP-Verlag $\mathrm{GmbH}, 1985$. 
Scheidegger, Christoph. "Hexen bei Zwingli." In Reformierte Kirche des Kanton Zürich. https://www.zhref.ch/themen/reformationsjubilaeum/allgemeine-informationen/huldrych-zwingli/zwingli-lexikon-von-a-bis-z1/lexikon-h/hexen.

Schlegel, Friedrich. Briefe an seinen Bruder August Wilhelm, edited by Oskar F. Walzel. Berlin: Verlag Speyer \& Peters, 1890.

Schutt, Christian, ed. Chronik der Schweiz. Zürich: Ex Libris Verlag / Chronik Verlag, 1987.

Sönke, Lorenz, and H.C. Erik Midelfort."Hexen und Hexenprozesse. Ein historischer Überblick, historicum.net, Mai 15, 2018. https://www.historicum.net/purl/aj/17.5.2017.

Withe, Hayden. "Fikcjonalność przedstawień opartych na faktach." Translated by Dorota Kołodziejczyk. In Withe, Hayden. Proza historyczna, edited by Ewa Domańska, 87-105. Kraków: Universitas, 2009.

\title{
,Weises' Lachen als Erziehungsmittel. Ricarda Huchs Novelle Teufeleien
}

\begin{abstract}
Der vorliegende Beitrag ist ein Versuch auf die wesentlichen Interessenfelder der frühen Schaffensphase von Ricarda Huch einzugehen. Einen methodologischen Zugriff bildet ihre Biografie sowie ihre geistesgeschichtliche Verankerung in der Epoche der deutschen Frühromantik. Im Sinne der Frühromantiker, vor allem Novalis', strebte Huch eine Synthese an zwischen den geschichtlich überlieferten Tatsachen und der Vorstellungswelt des dichtenden Künstlers, der mit seiner Dichtkunst ein pädagogisches Ziel verfolgt, dessen oberstes Prinzip das humane Verhalten des Menschen in der Gesellschaft sein soll.
\end{abstract}

Schlüsselwörter: Ricarda Huch, Historismus, Frühromantik, Reformation, Hexenverfolgungen, Humanismus.

\section{„Mądry” śmiech jako środek wychowawczy. Nowela Ricardy Huch Teufeleien}

\begin{abstract}
Abstrakt: Artykuł stanowi próbę przedstawienia najistotniejszych cech pisarstwa Ricardy Huch z jej wczesnej fazy twórczości. Z metodologicznego punktu widzenia podstawę do badań stanowią: biografia autorki oraz jej zakorzenienie w historii myśli wczesnego romantyzmu niemieckiego. W myśl wczesnych romantyków, a w szczególności Novalisa, Huch dążyła do swoistej syntezy między faktami historycznymi a sposobem postrzegania rzeczywistości przez piszącego artystę - ten jednocześnie myśląc o jemu współczesnych, kieruje się zamysłem pedagogicznym, którego nadrzędnym celem jest przestrzeganie zasad humanitaryzmu w społeczeństwie.
\end{abstract}

Słowa kluczowe: Ricarda Huch, historycyzm, wczesny romantyzm, reformacja, prześladowanie czarownic, humanitaryzm. 